\title{
IUPAC Technical Report "Metrological traceability of measurement results in chemistry: Concepts and implementation" available now
}

In recent years, the concept "traceability" in chemical measurement has received an extraordinary amount of attention. Still, the interpretation of "metrological traceability" varies in the literature. Now, the above-mentioned IUPAC Technical Report authored by Paul De Bièvre, René Dybkaer, Aleš Fajgelj and D. Brynn Hibbert appeared in Pure Appl. Chem. [doi: 10.1351/PAC-REP-07-09-39] and provides guidance in establishing and presenting metrological traceability of measurement results in chemistry. The Report builds on the 3rd edition of the International Vocabulary of Metrology (VIM 2008) where generic concepts for measurement are defined.

Establishing metrological traceability of measurement results is a prerequisite to obtaining their metrological comparability and to assessing their equivalence. In commerce, society, and science numerous comparisons of measurement results are performed daily. That requires a common, accepted definition of the concept "metrological comparability of measurement results" because many of these results are obtained at different locations and at different times. The Report presents the chemist with the metrological tools, procedures, and current vocabulary (VIM 2008) involved-it even defines the "metrological reference," something which was missing in the VIM 2008.

This IUPAC study aims at formulating recommendations and intends to provide the chemical measurement community with a consistent view of the creation, meaning, and role of metrological traceability and its underpinning concepts. A description is given of the calibration hierarchies needed in different circumstances to arrive at metrological traceability along a metrological traceability chain. Flow charts of generic calibration hierarchies are presented as well as a variety of examples. The establishment, assessment, and reporting of metrological traceability are discussed, including the provision of metrological references by a metrological institutional framework and the role of interlaboratory comparisons.

(Source: http://www.iupac.org/publications/pac/asap/ PAC-REP-07-09-39/). 